\title{
CORRIGENDA
}

\section{Linkage and association analysis of CACNG3 in childhood absence epilepsy}

\author{
Kate Everett, Barry Chioza, Jean Aicardi, Harald Aschauer, Oebele Brouwer, \\ Petra Callenbach, Athanasios Covanis, Olivier Dulac, Orvar Eeg-Olofsson, \\ Martha Feucht, Mogens Friis, Françoise Goutieres, Renzo Guerrini, Armin Heils, \\ Marianne Kjeldsen, Anna-Elina Lehesjoki, Andrew Makoff, Rima Nabbout, \\ Ingrid Olsson, Thomas Sander, Auli Sirén, Paul McKeigue, Robert Robinson, \\ Nichole Taske, Michele Rees and Mark Gardiner
}

European Journal of Human Genetics (2008) 16, 659-660; doi:10.1038/ejhg.2008.1

Correction to: European Journal of Human Genetics (2007) 15, 463-472; doi:10.1038/sj.ejhg.5201783; published online 31 January 2007

Due to minor changes in the clinical information, which have come to our attention, the data have been re-analysed. The corrected results for the linkage analysis are as follows: $\mathrm{HLOD}_{\max }=3.14(\alpha=0.6) ; \mathrm{NPL}_{\max }=2.68(P<0.003)$.

Twenty-three tag SNPs were used for initial association analysis. Of these, SNPs 3, 7 and 8 showed evidence for transmission disequilibrium. These three SNPs still show significant evidence for transmission disequilibrium upon re-analysis although the values are slightly altered (Table 1).
A 'sliding window' approach was used for haplotype association analysis of SNPs 2-8 which were in linkage disequilibrium (LD). Table 2 shows the corrected data

Table 1 SNPs showing statistically significant disease association $(P \leq 0.01)$ in at least one PDT test statistic in the entire resource

\begin{tabular}{lcccccccc}
\hline & & & \multicolumn{3}{c}{ Sum PDT } & \multicolumn{2}{c}{ Ave PDT } \\
\multirow{2}{*}{ SNP } & Minor allele & Trans & Not trans & $Z$ & P-value & $Z$ & P-value \\
& & & & & & & & \\
3 & $2(\mathrm{G})$ & 291 & 250 & 2.45 & 0.014 & 2.00 & 0.046 \\
7 & 2 (G) & 261 & 219 & 2.56 & 0.011 & 2.24 & 0.025 \\
8 & 2 (A) & 279 & 232 & 2.81 & 0.005 & 2.46 & 0.014 \\
\hline
\end{tabular}

Table 2 SNP-based sliding-window analysis of Block 1 showing windows which demonstrated significant $(P<0.05)$ global transmission disequilibrium in the entire resource when analysed using the PDT

\begin{tabular}{|c|c|c|c|c|c|c|c|c|c|c|c|c|c|c|c|c|c|}
\hline \multirow{2}{*}{$\begin{array}{l}S N P \\
2\end{array}$} & \multirow[b]{2}{*}{3} & \multirow[b]{2}{*}{4} & \multirow[b]{2}{*}{5} & \multirow[b]{2}{*}{6} & \multirow[b]{2}{*}{7} & & \multirow[t]{2}{*}{$\begin{array}{l}\text { Frequency in } \\
\text { parents (\%) }\end{array}$} & \multirow[t]{2}{*}{ Transmitted } & \multirow[t]{2}{*}{$\begin{array}{l}\text { Not } \\
\text { transmitted }\end{array}$} & \multicolumn{2}{|c|}{ Sum PDT } & \multicolumn{2}{|c|}{ Global } & \multicolumn{2}{|c|}{ AVE PDT } & \multicolumn{2}{|c|}{ Global } \\
\hline & & & & & & 8 & & & & $Z_{\text {(1d.f.) }}$ & P-value & $\chi_{\text {(d.f.) }}^{2}$ & P-value & $Z_{\text {(1d.f.) }}$ & P-value & $\chi_{\text {(d.f.) }}^{2}$ & P-value \\
\hline 2 & 2 & & & & & & 31.5 & 202 & 157 & 2.34 & 0.020 & 9.49 & 0.024 & 2.69 & 0.007 & $9.39_{(3)}$ & 0.025 \\
\hline 2 & 2 & 1 & & & & & 30.2 & 180 & 140 & 2.16 & 0.031 & $16.51_{(7)}$ & 0.021 & 2.50 & 0.013 & $14.59(7)$ & 0.042 \\
\hline & 2 & 1 & & & & & 44.1 & 255 & 214 & 2.51 & 0.012 & $9.39_{(3)}$ & 0.025 & 2.27 & 0.023 & $7.13_{(3)}$ & 0.068 \\
\hline & 2 & 1 & 1 & & & & 40.0 & 236 & 192 & 2.68 & 0.007 & $12.66_{(6)}$ & 0.049 & 2.46 & 0.014 & $9.74(6)$ & 0.136 \\
\hline & & 1 & 1 & & & & 48.4 & 27 & 228 & 2.53 & 0.011 & $9.93(3)$ & 0.019 & 2.22 & 0.026 & $7.24_{(3)}$ & 0.065 \\
\hline & & 1 & 1 & 1 & & & 42.2 & 215 & 170 & 2.86 & 0.004 & $14.24_{(6)}$ & 0.027 & 2.78 & 0.006 & $13.58_{(6)}$ & 0.035 \\
\hline & & & 1 & 1 & 2 & & 36.8 & 203 & 155 & 3.07 & 0.002 & $20.95_{(7)}$ & 0.004 & 3.02 & 0.003 & $20.79_{(7)}$ & 0.004 \\
\hline & & & 1 & 1 & 2 & 2 & 36.3 & 199 & 148 & 3.29 & 0.001 & $27.69(12)$ & 0.006 & 3.33 & 0.001 & $27.62_{(12)}$ & 0.006 \\
\hline & & & & 1 & 2 & & 40.2 & 218 & 171 & 3.02 & 0.003 & $15.07_{(3)}$ & 0.002 & 2.95 & 0.003 & $14.86_{(3)}$ & 0.002 \\
\hline & & & & 1 & 2 & 2 & 39.4 & 215 & 159 & 3.74 & 0.000 & $27.77_{(6)}$ & 0.000 & 3.49 & 0.001 & $25.24_{(6)}$ & 0.000 \\
\hline & & & & & 2 & 2 & 45.8 & 27 & 218 & 3.06 & 0.002 & $16.16_{(3)}$ & 0.001 & 2.95 & 0.003 & $14.88_{(3)}$ & 0.002 \\
\hline
\end{tabular}

Only haplotypes showing significant $(P<0.05)$ overtransmission are shown. 
for this analysis. As in the original analysis, no single complete haplotype within the LD block was sufficiently common to allow demonstration of disease association on the global level. However, using the sliding window approach, associated haplotypes were identified composed of combinations of SNPs 2-8. The individual haplotypes which are overtransmitted within each window together form a larger haplotype composed of the alleles 2211122.

While subtle differences have been found in this reanalysis, this was not found to alter the conclusions drawn previously.

\section{Unexpected genetic heterogeneity in a large consanguineous Brazilian pedigree presenting deafness}

Karina Lezirovitz, Eliete Pardono, Maria TB de Mello Auricchio, Fernando L de Carvalho e Silva, Juliana J Lopes, Ronaldo S Abreu-Silva, Jihane Romanos, Ana C Batissoco and Regina C Mingroni-Netto

European Journal of Human Genetics (2008) 16, 660; doi:10.1038/ejhg.2008.2

Correction to: European Journal of Human Genetics (2008) 16, 89-96; doi:10.1038/sj.ejhg.5201917; published online 12 September 2007
Since the publication of the above paper, the authors have identified three typographical errors regarding Table 1 . The amended table is shown below.

Table 1 Summary description of the genotypic data

\begin{tabular}{|c|c|c|}
\hline Genotypic data & $\begin{array}{l}\text { Number of } \\
\text { individuals }\end{array}$ & Pedigree position \\
\hline MY015A mutations in both alleles & 20 & \\
\hline c.10573delA homozygotes & 15 & $\begin{array}{l}\text { V:8, V:12, V:18, V:22, V:23, V:24, V:25, V:27,V:34, Vl:2, } \\
\text { VI:3, Vl:4, VI:8, Vl:9 and Vl:11 }\end{array}$ \\
\hline $\begin{array}{l}\text { c. } 10573 \text { delA/c.9957_9960delTGAC compound } \\
\text { heterozygotes }\end{array}$ & 5 & $\mathrm{~V}: 1, \mathrm{~V}: 2, \mathrm{~V}: 3, \mathrm{~V}: 4$ and $\mathrm{VII}: 2$ \\
\hline Unsolved cases & 6 & \\
\hline One MYO15A mutation detected & 1 & $\mathrm{~V}: 17$ \\
\hline No MYO15A mutations & 5 & VI:17, VI:19, VII:4, VII:3 and VIII:1 \\
\hline
\end{tabular}

